\title{
Open
}

Review

\section{Regulatory networks between neurotrophins and miRNAs in brain diseases and cancers}

\author{
Jian $\mathrm{SHI}^{*}$ \\ Department of Neurology, Dept of Veterans Affairs Medical Center, San Francisco and University of California, San Francisco, CA \\ 94121, USA
}

\begin{abstract}
Neurotrophins are involved in many physiological and pathological processes in the nervous system. They regulate and modify signal transduction, transcription and translation in neurons. It is recently demonstrated that the neurotrophin expression is regulated by microRNAs (miRNAs), changing our views on neurotrophins and miRNAs. Generally, miRNAs regulate neurotrophins and their receptors in at least two ways: (1) miRNAs bind directly to the $3^{\prime}$ untranslated region (UTR) of isoform-specific mRNAs and post-transcriptionally regulate their expression; (2) miRNAs bind to the $3^{\prime}$ UTR of the regulatory factors of neurotrophins and regulate their expression. On the other hand, neurotrophins can regulate miRNAs. The results of BNDF research show that neurotrophins regulate miRNAs in at least three ways: (1) ERK stimulation enhances the activation of TRBP (HIV-1 TAR RNA-binding protein) and Dicer, leading to the upregulation of miRNA biogenesis; (2) ERK-dependent upregulation of Lin28a (RNA-binding proteins) blocks select miRNA biogenesis; (3) transcriptional regulation of miRNA expression through activation of transcription factors, including CREB and NF-KB. These regulatory processes integrate positive and negative regulatory loops in neurotrophin and miRNA signaling pathways, and also expand the function of neurotrophins and miRNAs. In this review, we summarize the current knowledge of the regulatory networks between neurotrophins and miRNAs in brain diseases and cancers, for which novel cutting edge therapeutic, delivery and diagnostic approaches are emerging.
\end{abstract}

Keywords: neurotrophin; miRNA; BNDF; ERK; CREB; NF-kB; brain injury; neurodegenerative disease; cancer

Acta Pharmacologica Sinica (2015) 36: 149-157; doi: 10.1038/aps.2014.135; published online 29 Dec 2014

\section{Introduction}

The neurotrophin family of growth factors includes nerve growth factor (NGF), brain-derived neurotrophic factor (BDNF), neurotrophin 3 (NT3) and neurotrophin 4 (NT4), which are important for both physiological and pathological processes $^{[1-5]}$. There are two types of neurotrophin receptors: the low-affinity p75 neurotrophin receptor (NTR) and highaffinity tropomyosin-related kinase (TRK) receptors, including TRKA, TRKB and TRKC. All four neurotrophins may stimulate cells via p75NTR. NGF, BDNF, and NT4, and NT3 also act via TRKA, TRKB, and TRKC respectively. NT3 binds most strongly to TRKC. However, under some circumstances it may also act via TRKA and TRKB. Several intracellular signaling pathways are activated by the binding of neurotrophins to TRK receptors, including the extracellular-signal-regulated kinase 1/2 (ERK1/2), phosphoinositide 3-kinase (PI3K), and phospholipase C (PLC) pathways ${ }^{[1,2]}$. The signaling pathways

\footnotetext{
* To whom correspondence should be addressed.

E-mail Jian.shi@ucsf.edu

Received 2014-09-04 Accepted 2014-11-14
}

activated through p75NTR are more complicated and unclear, and several reviews discuss these pathways ${ }^{[6,7]}$. In addition, p75NTR has been implicated as a direct regulator of neurogenesis $^{[8,9]}$, and the increase in adult neurogenesis by NGF and its mimic may go through p75NTR pathways ${ }^{[10,11]}$. Although these pathways activate many effects of neurotrophins, their most important function is to regulate specific changes in gene transcription. Neurotrophins can directly regulate the expression and activation of transcription factors, including cAMP-response element binding protein (CREB) and nuclear factor kappaB (NF-kB). Although neurotrophin stimulation can affect the expression of hundreds of genes, the networks between neurotrophins, transcription factors and their target genes are not fully understood. To date, most studies have focused on the neurotrophin-induced expression of proteinencoding genes. However, miRNAs are also transcriptionally induced by neurotrophins ${ }^{[12,13]}$.

MicroRNAs (miRNAs) are 20-22 nucleotide non-coding RNA molecules, which regulate gene expression at the posttranscriptional level and have changed our evolutionary view of gene regulation ${ }^{[14,15]}$. MiRNA genes are usually located in 
intergenic or intronic regions as individual or clustered genes. Several steps must occur before miRNAs can function, including transcription of primary miRNAs (pri-miRNAs), formation of precursor miRNAs (pre-miRNAs), pre-miRNA transportation out of the nucleus, and miRNA maturation. Many enzymes, including RNA polymerase II, Drosha, Exportin 5, Dicer and Argonaute (Ago), are involved in these processes, and more details can be found in several reviews ${ }^{[14-16]}$. The mature miRNAs remain in the Ago complex, and the antisense miRNAs (miRNAs*) are mostly degraded ${ }^{[12]}$. MiRNAs target mRNAs by preferentially binding to their $3^{\prime}$ untranslated regions ( $\left.3^{\prime} \mathrm{UTR}\right)$, promoting their degradation or suppressing their translation into proteins, thereby silencing genes. A given miRNA can target approximately a hundred mRNAs, each of which has multiple binding sites, enhancing the overall effects. An mRNA can be targeted by dozens of miRNAs, thereby responding to different functions. However, approximately $30 \%$ of the human protein-coding genome is targeted by miRNAs ${ }^{[17]}$. Some mRNA transcripts contain a short $3^{\prime}$ UTR and lack a miRNA binding domain. Many of these 'short' mRNAs regulate key cellular functions such as DNA repair and proliferation ${ }^{[18]}$.

The complex network of miRNAs and the regulation of gene expression by miRNAs are not fully understood, although miRNA biogenesis and basic functions have been known for several years. Knowledge remains scarce about the upstream regulators of miRNAs that manage their transcriptional and post-transcriptional control, although thousands of miRNAs have been discovered. A new era of miRNA regulation is occurring in neurotrophin studies, although these too have already been studied for decades. Although this review is focused on the regulation between neurotrophins and miRNAs in cancer and brain diseases, this regulation is difficult to clarify due to the triangular, nonlinear relationship between neurotrophins and miRNAs. Neurotrophins can regulate the transcription of a subset of miRNAs and transcription factors, resulting in complex regulatory circuits involving positive and negative feedback loops (Figure 1). For this review, "neurotrophins" and "miRNAs" were used as keywords to search the MEDLINE electronic database for English-language articles reported between January 1994 and May 2014. Relevant articles were then scanned for additional studies.

\section{Regulation of neurotrophins by miRNAs}

Brain specific miRNAs were found to play important roles in neurotrophin expression soon after the recognition of miRNAs as fundamental regulators of gene expression. Because neurotrophins such as BDNF are key players in many neuronal functions including survival, synaptic plasticity and neurogenesis in the central nervous system (CNS), miRNAs should also be involved in most neurotrophin effects. Indeed, the interaction between BDNF and brain-specific miRNAs is an area of recent focus. Dicer1 is the key enzyme that processes pre-miRNA nucleotide duplexes. Many miRNAs were significantly decreased after Dicer1 was deleted in the mouse forebrain, including miR-124, miR-132, miR-137, miR-138, miR-

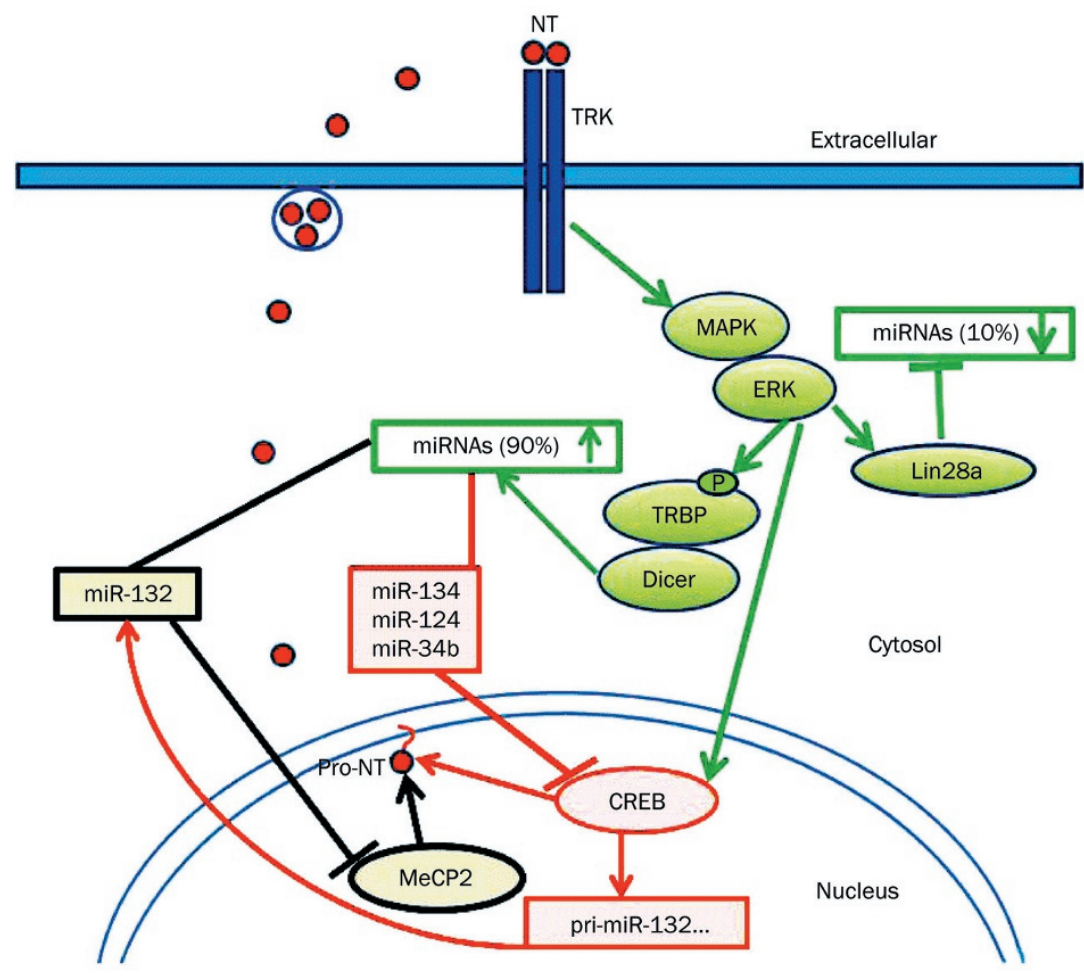

Figure 1. The regulatory loops of neurotrophins (NTs), miRNAs and transcription factors. Green represents NT regulation of miRNAs through ERKTREP/Dicer, ERK-Lin28a, and ERK-CREB. Red denotes miRNA regulation of NTs by post-transcriptionally inhibiting CREB expression. Black shows the regulatory loop of NT (BDNF), MeCP2, and miR-132 that exists in brain diseases including Rett Syndrome, ischemia, HD and PD. 
29a, and miR-29c. Interestingly, the expression of BDNF and pro-BDNF were higher in Dicer1-deficient mice compared to controls $^{[19]}$. This suggested that BDNF expression was suppressed by miRNAs in wild-type animals. Consistent with the Dicer1 deficiency results, studies of miR-132 and miR-182 support their roles as negative regulators of BDNF in human neuronal cells. Lower BDNF expression correlated with higher miR-132 and miR-182 expression in the serum of depressive patients ${ }^{[20]}$. However, it is unclear how these miRNAs affect BDNF expression. The mechanism by which miR-134 regulates BDNF may provide a clue. MiR-134 regulates BDNF expression by post-transcriptionally regulating CREB expression. The transcription factor CREB recruits its co-activators CREB-binding protein (CBP) and p300 into a transcriptional complex, activating CREB-dependent transcription ${ }^{[21]}$. CREB also mediates the transcription of BDNF. CREB can bind to several BDNF promoter elements and enhance its activitydependent regulation ${ }^{[22]}$. However, there are three partially complementary binding sites for miR-134 in the 3'UTR of the mouse CREB gene. One site is well-conserved, and miR-134 can bind directly to it and inhibit the CREB translation. CREB inhibition will therefore inhibit BDNF gene expression. CREB, BDNF and miR-134 therefore comprise a regulatory loop (Figure 1). Consistently, BDNF and CREB are both downregulated when miR-134 is up-regulated in SIRT1 $\Delta$ mice $^{[23]}$.

NGF, NT3, TrkA, and p75NTR are also regulated by miRNAs in certain conditions. P75NTR is up-regulated in brain injuries and neurodegenerative diseases and is involved in apoptosis. The underlying mechanism of p75NTR upregulation is still unclear ${ }^{[10,24]}$. Recently, it was reported that p75NTR expression is regulated by miR-592, and there is an inverse correlation between miR-592 and p75NTR levels. After cerebral ischemia, miR-592 levels dramatically decreased. In contrast, the p75NTR level significantly increased. There is a conserved miR-592 binding site in the 3'UTR of p75NTR. Importantly, miR-592 over-expression decreased the level of p75NTR, and in contrast, transduced anti-miR-592 caused a significant increase in the p75NTR protein level ${ }^{[25]}$. A miR-21 binding site was identified in the NT3 3'UTR, and there was an inverse relationship between NT3 and miR-21 in cultured hippocampal neurons. Further, in the epileptic model, NT3 mRNA levels decreased in the hippocampus following miR21 increase. Notably, miR-21 is a candidate for regulating NT3 signaling in the hippocampus following status epileptics ${ }^{[26]}$. Likewise, miR-221, a candidate regulator of NGF and TRKA, is highly conserved in vertebrates. Recently, it was reported that miR-221 could suppress NGF and TRKA at both the protein and mRNA levels. In respiratory syncytial virus (RSV) infected airways, the expression of NGF and TRKA were upregulated by silencing miR-221 expression in the infected cells, and this benefited viral production ${ }^{[27]}$.

MiRNAs regulate neurotrophins and their receptors in an isoform-specific way. There are two BDNF mRNA isoforms that translate to the same BDNF protein. Sequence analysis revealed that there were three miR-206 target sites in the long BDNF 3'UTR (BDNF-L), but only one in the short BDNF 3'UTR
(BDNF-S). Transfection of pre-miR-206 repressed endogenous BDNF mRNA levels, and the suppressive effect was related to the miR-206 targeting sites in the BDNF-L 3'UTR but not the site in the BDNF-S ${ }^{[28]}$. Similarly, TRKC has two major protein isoforms detected in human brain samples: the fulllength, kinase-active receptor (FL-TRKC) and a truncated noncatalytic isoform (T-TRKC). Different functions were found for T-TRKC, apart from ligand competition with full-length receptors. FL-TRKC levels were significantly reduced by miR-151-3p alone. Significant T-TRKC downregulation was observed with the over-expression of miR-128, miR-485-3p, miR-765, and miR-768-5 $p^{[29]}$. Importantly, the overexpression of miR-128 and the inhibition of T-TRKC had the same effects, including increased cell number. Different miRNA regulation of different mRNA isoforms therefore mediates different functions in different situations.

\section{Regulation of miRNAs by neurotrophins}

BDNF may widely regulate miRNA biosynthesis by enhancing the function of Dicer, the general miRNA processing enzyme, via the MAPK/ERK pathway ${ }^{[30]}$. To enhance Dicer function, BDNF activates the MAPK/ERK signaling pathway through the TRKB receptors. Dicer and phospho-TRBP (HIV-1 TAR RNA-binding protein) comprise the miRNA-generating complex. TRBP phosphorylation and Dicer expression are mediated by ERK. The phosphorylation of TRBP enhances miRNA production by increasing the stability of the miRNAgenerating complex with Dicer ${ }^{[31]}$. BDNF rapidly elevates Dicer, stabilizes the complex, and increases the levels of mature miRNAs. Remarkably, approximately $89.4 \%$ of detectable endogenous miRNAs were increased more than 2-fold by BDNF, whereas only $10.6 \%$ decreased to $<50 \%{ }^{[32]}$ (Figure 1 ). In addition to the regulatory steps described above, miRNA biogenesis can be regulated at multiple steps by other transacting factors, including the Lin28 RNA-binding proteins ${ }^{[33]}$. Moreover, some BDNF-inhibited miRNAs that contain Lin28a binding sites in their terminal loop region were suppressed in their processing from pre-miRNA to mature miRNA by Lin28a ${ }^{[33]}$ when Lin28a was elevated by BDNF. This could provide a mechanism for decreasing the levels of select mature miRNAs even in the context of Dicer elevation ${ }^{[32]}$. These findings are not only consistent with transcriptional specificity in response to BDNF but also with the increased knowledge of the regulatory processes of BDNF (Figure 1). This transcriptional regulation affects the functions of BDNF. For example, targets that are usually upregulated by BDNF, including AMPA glutamate receptor subunit GluA1 (GluA1), calcium calmodulindependent protein kinase II (CaMKIIa), and Homer2, failed to be upregulated by BDNF in Dicer-deficient neurons. A target normally downregulated by BDNF, the potassium-chloride cotransporter (KCC2), was nonresponsive to BDNF in Dicerdeficient neurons ${ }^{[32]}$. Taken together, at least three ERK related regulatory pathways function in BDNF regulated miRNA biogenesis: (1) the ERK dependent enhancement of TRBP and Dicer upregulates miRNA biogenesis; (2) the ERK dependent upregulation of Lin28a downregulates select miRNA expres- 
sion; (3) the activation of specific transcription factors, such as CREB, by BDNF. NGF and NT3 also can trigger the PI3K/ AKT and MAPK/ERK signaling pathways through TRKA and TRKC ${ }^{[1,2]}$. The question is whether NGF or NT3 can enhance miRNA expression by enhancing ERK and Dicer in TRKA or TRKC positive cells.

Neurotrophins could also regulate the expression of miRNAs by regulating specific transcription factors, including CREB and NF-KB. The mechanism of miRNA transcription is the same as that of mRNAs, in which transcription factors play important roles. Neurotrophins regulate CREB activation through TRK receptors ${ }^{[1,2]}$, so neurotrophins may regulate miRNA expression by activating specific transcription factors. Computational searches revealed that several miRNAs, including miR-124a, miR-9, miR-29a/29b, and miR-132, contain a conserved CRE site in their promoters ${ }^{[34]}$. The ERK1/2 dependent pathways regulating miR-212/132 discussed above also have CREB dependent and independent aspects. The miR-212/132 cluster had absolute fold changes $>4$ after BDNF treatment in cultured primary cortical mouse neurons. Some of the pri-miR-212/132 transcription in response to BDNF was dependent on the activation of ERK1/2 and Dicer, but others were dependent on the activation of ERK1/2 and CREB ${ }^{[35]}$ (Figure 1). In the CREB dependent pathway, the phosphorylation of CREB by MSKs downstream of ERK1/2 was involved in pri-miR-212/132 transcription. CREB binding sites have been shown previously in the miR-212/132 and miR-9 loci. Importantly, recent studies demonstrated experimentally that miR-212/132 and miR-9 are regulated by CREB and involved in regulating neuronal morphogenesis ${ }^{[36-38]}$. Similarly, NF-KB can be regulated by p75NTR signaling ${ }^{[6]}$. Using selective NF-KB translocation and DNA binding inhibitors, miR-9, miR$125 \mathrm{~b}$, and miR-146a up-regulation was shown to be sensitive to NF- $\mathrm{KB}^{[39]}$. Consistent with this result, the expression of miR$146 \mathrm{a}$ was proved to be regulated by the p65 subunit of NF-KB in a Huntington cellular model ${ }^{[40]}$. Additionally, miR-21 was regulated by NF-KB and involved in the apoptosis of human myeloma cells ${ }^{[41]}$.

NGF induction of miRNA expression also depends on the activation of the ERK1/2 and CREB pathway. For example, the expression of miR-221 and miR-222 were induced by NGF stimulation in PC12 cells. This induction was ERK related, and these miRNAs might contribute to the mechanism of NGF related cell survival in PC12 cells. In fact, miR-221 and miR-222 are growth related and derived from the same pri$\operatorname{miRNA}^{[42]}$. However, it is unclear whether NGF enhances the activation of Dicer after sustained ERK activation. Other PC12 studies showed that additional miRNAs were modulated by NGF, including miR-21, miR-29c, miR-30c, miR-93, miR103, miR-207, miR-691, and miR-709. Among these miRNAs, significant CRE binding enrichment was identified in the promoter area, indicating that NGF regulated those miRNAs through the ERK and CREB pathway ${ }^{[43]}$. Notably, another study showed that NGF treatment increased the expression of miR-181a, miR-221 and miR-326 and decreased the expression of miR-106b, miR-126, miR-139-3p, miR-143, miR-210, and
miR-532-3p. MiR-221 was dramatically up-regulated ${ }^{[44]}$. Interestingly, the authors of this paper found a Lin28a binding site in several miRNAs, including miR-139, mir-143 ${ }^{[33]}$ and miR532 , after analyzing the terminal loop region of those miRNAs inhibited by NGF. NGF may downregulate those miRNAs by the same mechanism as BDNF, enhancing Lin28a through activated ERK signaling. The neurotrophin regulation of miRNA synthesis widely expands the functional network of neurotrophins, as one miRNA can regulate approximately one hundred mRNAs.

\section{The effects of neurotrophins and miRNAs in brain diseases}

Rett Syndrome and Huntington's disease (HD) may have a similar regulatory loop mechanism of miR-132, methyl-CpG binding protein 2 (MeCP2) and BDNF. MeCP2 gene mutations cause Rett syndrome, a progressive neurologic developmental disorder. Interestingly, MeCP2 overexpression increased BDNF transcript levels, which consequently resulted in the activation of miR-132 through ERK dependent pathways. Increased miR-132 led to a decrease in the MeCP2 levels ${ }^{[45]}$. This suggests a negative regulatory loop exists among miR132, BDNF, and MeCP2, shown in Figure 1. To support this notion, experimentally blocking miR-132 led to increased levels of MeCP2 and BDNF, and loss of MeCP2 decreased BDNF and miR-132 ${ }^{[46]}$. In some Rett syndrome patients, miR-132 may be abnormally upregulated, causing low levels of MeCP2 and, in turn, leading to disease. In Huntington disease, the regulatory loop of miR-132, BDNF, and MeCP2 also exists. MiRNA expression analysis revealed that miR-9/9*, miR29b, and miR-124 were downregulated in HD patient brains, whereas miR-29a and miR-132 were up-regulated ${ }^{[47,48]}$. However, a contradictory report showed decreased miR-132 in HD animal models, which may be caused by the different disease stages $^{[48]}$. Upregulated miR-132 may suppress the expression of MeCP2 and then suppress the expression of BDNF. Suppressed expression of BDNF in striatal afferents may cause the neuronal death and behavioral dysfunction of HD. It is not clear that how the progressive loss of BDNF in cortical or subcortical afferents contributes to striatal atrophy and behavioral dysfunction in HD patients. However, we can find some clues from HD animal studies. In R6/2 transgenic mice, a HD animal model, decreased BDNF expression in the motor cortex and thalamic afferents started at 6 weeks and progressed until death at 13-15 weeks, coinciding with striatal neuronal loss and behavior dysfunction ${ }^{[49]}$. In addition, BDNF protein levels were significantly decreased in the striatum of Mecp2-deficient mice, which was caused by a BDNF trafficking dysfunction ${ }^{[50]}$. Therefore, miR-132, BDNF, and MeCP2 are all involved in the regulatory mechanism of Rett Syndrome and HD.

The regulatory loop of miR-132, BDNF, and MeCP2, as well as other miRNAs and factors, exists in Parkinson disease (PD). Several miRNAs were reported in epigenetic alterations of PD, including miR-132, miR-184, and miR-34a. A feedback loop between epigenetic mechanisms and miR-132, which may act as mediators of $\mathrm{PD}$, was established via its repressive effect 
on $\mathrm{MeCP} 2$ and silent information regulator 1 (SIRT1) ${ }^{[51]}$. In a PD animal model, microarray and $\mathrm{qPCR}$ results confirmed a significant increase in miR-132 in affected rats. Nuclear receptor related 1 protein (Nurr1) is one of the known downstream targets of miR-132, and Nurr1 is essential in the neurogenesis of midbrain dopaminergic neurons. In PD rats, significant decreases in Nurr1 and BDNF expression in the mesencephalic neurons were observed, and BDNF is a direct target of Nurr1 ${ }^{[52]}$. Unlike miR-132, miR-184 is a target of MeCP2. The expression of miR-184, a brain-specific miRNA, is repressed by the binding of MeCP2 to its promoter, inducing methylationspecific transcriptional repression. This relationship links DNA methylation to miR-184 activity, and this regulation affected synaptic plasticity ${ }^{[53]}$. The leucine-rich repeat kinase 2 LRRK2 causes familial and sporadic PD. Increasing the level of miR-184* (antisense miR-184) attenuated the pathogenic LRRK2 effects in Drosophila ${ }^{[54]}$. These findings suggest that miR-184*, MeCP2, and LRRK2 are related to PD. MiR-184*, but not miR-184, acted on its targets directly, although most antisense miRNAs are predicted to be degraded after synthesis. Similar to miR-132, miR-34a also inhibited SIRT1, a gene that regulates cellular senescence, limited cellular longevity and increased apoptosi ${ }^{[55]}$. In addition, as a miR-34a target, CREB plays an important role in PD. CREB is necessary for the anchoring of CREB-binding protein (CBP), which contains a histone acetyltransferase (HAT) domain, and in turn co-activates CREB to exert its beneficial function on neuronal plasticity $^{[51]}$. The suppression of CREB expression by miR-34a may also indirectly suppress BDNF expression and increase neuronal apoptosis. Here, miR-132, miR-184*, and miR-34a promote the epigenetic alterations of PD via DNA methylation, histone acetylation and the expression of BDNF and other factors.

Neurotrophins and miRNAs regulate cell survival and function in brain injuries and diseases. The p75NTR expression levels in neurons are partly related to the expression of miR592, and there is an inverse correlation between miR-592 and p75NTR levels in the adult brain. In cerebral ischemia, an increase in p75NTR levels correlated with decreased miR-592 levels. Importantly, overexpression of miR-592 in neurons decreased p75NTR expression levels and attenuated the activation of pro-apoptotic signaling and cell death induced by ischemic injury. These results suggest that miR-592, a key regulator of p75NTR expression and a suppressor of neuronal apoptosis, is a potential therapeutic candidate for ischemic injury $^{[25]}$. MiR-132 and MeCP2 were involved in a preconditioning ischemia study. Downregulation of miR-132 in preconditioning ischemia induced a rapid increase in MeCP2 protein in the mouse cortex. This increased the ischemic tolerance. BDNF expression may be increased by this upregulation of MeCP2 expression, and this is supposed to be the underlying mechanism of ischemic tolerance. These studies suggest that miR-132, MeCP2, and BDNF could serve as effectors of ischemic preconditioning-induced tolerance ${ }^{[56]}$. In addition, many overloaded miRNAs were found in Aicardi-Goutiéres syndrome (AGS) patients, whose symptoms include calcification of the basal ganglia, abnormal cerebral white matter and diffuse brain atrophy ${ }^{[57]}$. The genetic pathological mechanisms of AGS show that inherited or sporadic AGS mutations downregulate RNAse expression, thus inducing overload of many miRNAs, which in turn activate neurotoxic lymphocytes and inhibit angiogenesis. Interestingly, AGS caused more than 16-fold up-regulation of miR-134 ${ }^{[57]}$, which targets and decreases BDNF expression, thereby potentially causing neuronal apoptosis and brain atrophy.

In a neurodegenerative cellular model, withdrawing NGF causes neuronal differentiation dysfunction and cell apoptosis. Because miR-21 enhanced neurotrophin signaling and controlled the neuronal differentiation induced by NGF, this miRNA preserved the neurite network and supported the viability of the neurons after NGF withdrawal ${ }^{[43]}$. MiR-21 also regulates the expression of NT3 in hippocampal neurons of status epileptic brains. There is a miR-21 binding site in the 3'UTR of NT3. In several epilepsy models, NT3 mRNA levels decreased consistently with decreased neurite outgrowth and neuronal cell survival. Recently, it was found that miR-21 expression levels inversely correlated with NT3 mRNA levels in hippocampal neuronal cultures. To mimic the episode of status epileptics, treatment with excess $\mathrm{KCl}$, a depolarizing agent in the neurons, also resulted in an increase in miR-21 and a decrease in NT3 mRNA. MiR-21 may also be a candidate for regulating NT3 signaling in the rat hippocampus following status epileptics ${ }^{[26]}$. Moreover, in a degenerative cellular model of Alzheimer's disease, primary cultured neurons incubated with the amyloid beta 25-35 $\left(\mathrm{A} \beta_{25-35}\right)$, pretreatment with neuropeptide Y (NPY) decreased miR-30a-5p and increased BDNF mRNA and protein levels. Accordingly, miR-30a-5p targeted the 3'UTR of BDNF and down-regulated BDNF protein levels in human neuronal cells ${ }^{[58]}$. Thus, the miR-30a-5p and BDNF alterations were present in AD-affected neurons. NPY, by influencing miRNA expression, may affect BDNF production and exert neuronal protective effects ${ }^{[59]}$.

\section{The effects of neurotrophins and miRNAs in cancers}

Neurotrophins and miRNAs are all involved in cancer. Similar to many other growth factors, neurotrophin signaling is involved in several cancers, and TRK receptor activation can either enhance or suppress tumor growth. The miRNA expression levels are altered in many cancers, resulting in abnormal increases or decreases. As post-transcriptional regulators, miRNAs can either act as oncogenes or tumor suppressor genes. The crosstalk between neurotrophins and miRNAs will be a very interesting topic in cancer studies for identifying novel diagnosis and therapeutic methods. For example, the BDNF/TRKB pathway has a critical role in tumorigenesis, promoting proliferation, differentiation, angiogenesis and invasiveness in several tumors, including neuroblastoma, ovarian, breast, prostate and lung cancers. Among these tumors, the overexpression of BDNF/TRKB might provide an autocrine survival pathway, and has also been implicated in poor prognosis in those tumors ${ }^{[60-63]}$. Interestingly, miR-204 has an equally important role in tumorigenesis, migration and invasion. In fact, the chromosomal locus containing miR-204 
is frequently lost, resulting in its lower expression in ovarian cancers, breast cancers and pediatric renal tumors. BDNF was predicted to be a miR-204 target from several prediction algorithms, and this was proved experimentally. Recently, it was reported that loss of miR-204 resulted in BDNF/TRKB overexpression and activation of the AKT/mTOR/Rac1 signaling pathway, leading to cancer cell migration and invasion ${ }^{[13]}$. Thus, miR-204 acts as a potent tumor growth and metastasis suppressor, inhibiting at least one of the BDNF-TRKB pathways. In addition to BDNF, NT3 can also activate TRKB signaling in some circumstances such as in breast cancers. Similarly, miR-200c is expressed in breast cancers, but is lost in the more aggressive triple negative breast cancers (TNBC). It is a potent repressor of the epithelial to mesenchymal transition that plays an important role in the ability of cells to acquire the traits necessary to metastasize. MiR-200c may serve as a potent modulator of subclass-specific gene expression networks in breast cancer, and may be a useful tool for the subclassification of breast cancers. Anoikis is a type of apoptosis that is triggered by cell detachment from the native extracellular matrix. In suspended anoikis resistant TNBC cells, the upregulation of both TRKB and NT3 suggests that they are necessary for survival in suspension. Importantly, miR-200c can directly target TRKB and NT3 to restore the anoikis sensitivity of TNBC cells ${ }^{[64]}$. In this study, NT3 was identified as a TRKB ligand capable of activating TRKB to induce anoikis resistance. Overexpression of miR-200c decreased both TRKB and NT3, thereby restoring anoikis sensitivity ${ }^{[64]}$. These results indicate that miRNAs, neurotrophins and their receptors comprise a possible therapeutic regimen to treat different types and stages of tumors, and can also be used as diagnostics for cancer detection and prediction.

MiRNAs regulate different TRKC isoforms in cancers. The TRKC gene can be alternatively spliced, yielding different isoforms. These include FL-TRKC and T-TRKC, which differ functionally and have separate $3^{\prime}$ UTRs. This structural difference supports the hypothesis that these two isoforms may be differentially regulated by distinct miRNAs. Interestingly, only the truncated TRKC isoform possesses the target sequences for miR-9, miR-125a, and miR-125b. Furthermore, overexpression of miR-9 and miR-125a/b suppressed the expression of the truncated TRKC receptor isoform and decreased the proliferation of SK-N-BE neuroblastoma cells ${ }^{[65]}$. In SH-SY5Y neuroblastoma cells, miR-151-3p alone repressed FL-TRKC. Four other miRNAs, including miR-128, miR485-3p, miR-765, and miR-768-5p, repressed T-TRKC. In particular, miR-128 overexpression caused the same morphological changes in SH-SY5Y neuroblastoma cells that were observed using a siRNA specifically against truncated TRKC, as well as a significant increase in the cell number ${ }^{[29]}$. The isoform-specific regulation of TRKC by miRNAs suggests that neurotrophins and their receptors can mediate processes that are strongly linked to miRNA-dependent mechanisms.

MiRNAs may be involved in the ligand-independent function of p75NTR in glioma. Some of the different functions stimulated by neurotrophins through their receptors appear to be ligand-independent. For example, several reports suggest a type of ligand-independent activation of p75NTR signaling. After the ligand binding site of p75NTR, Cys257, was deleted in the transmembrane domain, ligand-independent activity still occurred through several pathways that are normally activated by NGF binding to p75NTR ${ }^{[6]}$. MiRNAs are candidates to conduct the ligand independent function. Usually, p75NTR acts as a tumor suppressor, causing cellular apoptosis and suppressing metastatic invasion. However, p75NTR can also induce invasion and metastasis in glioma and melanoma. MiR-6165 resides in the 4th intron of p75NTR in both the human and rat genomes. However, an independent promoter for miR-6165 was not found, and its transcription was thought to occur through its host gene promoter, the p75NTR promoter. Endogenous miR-6165 is expressed together with p75NTR in several glioma cell lines and glioma primary tumors. Similar to the pro-apoptotic role of p75NTR, overexpression of pre-miR-6165 in U87, a human malignant glioma cell line, resulted in an elevated rate of apoptosis. MiR6165 functions on its target genes, including neural stem cellderived dendrite regulator (DAGLA) and Polycistin-1 (PKD1), but not on any p75NTR downstream pathways ${ }^{[6]}$. Therefore, the function of miR-6165 is p75NTR related but ligand independent.

The miRNA delivery system is one of the important issues in the treatment of cancer and brain diseases. Without protection, miRNAs or siRNAs will be degraded in less than $30 \mathrm{~min}$ in the circulatory system. To avoid this, nanomedicine and nanotechnology methods are being applied to deliver miRNAs. They comprise a novel research field, focusing on formulating biocompatible therapeutic agents such as nanoparticles, nanocapsules, micellar systems, and conjugates ${ }^{[68]}$. A nano-carrier system is required to deliver DNA or RNA to cells, which enhances cell internalization and protects the nucleic acid from nuclease enzymatic degradation. In addition, nanoparticles may also protect neurons from neurotoxicity due to overloaded nucleic acid ${ }^{[57,69]}$. Several nanoparticlebased delivery strategies have been developed in recent years, including poly ( $D L$-lactide-co-glycolide) (PLGA) nanoparticles, an FDA-approved commercial material and the most well-studied degradable synthetic polymer in humans. They are effective drug delivery vehicles ${ }^{[70]}$ and are particularly attractive carriers for delivering miRNAs and siRNAs. Most importantly, nanoparticles composed of PLGA loaded with a drug have recently been successfully delivered intranasally into the brain ${ }^{[71]}$, and PLGA loaded with a different drug has been delivered orally into the circulatory system ${ }^{[72]}$. PLGA nanoparticle-delivering siRNAs or miRNAs have been used widely in cancer ${ }^{[73,74]}$ as well as Alzheimer's disease in animal models ${ }^{[75]}$. Importantly, other chemicals, nucleic acids and proteins can be added to the PLGA matrix to extend the features of nanoparticles. The emergence of nanotechnology as a delivery mechanism trends toward clinical development of miRNA therapies in cancer and brain diseases. 


\section{Conclusion and outlook}

While the main focus of previous neurotrophin studies was on their function, signal transduction and regulation, since the beginning of the miRNA era, research has focused on fundamental processes, genomic alterations and target genes. Recently, studies have identified new regulation of miRNAs and neurotrophins such as miR-6165 and BDNF. They explored positive and negative loops among miRNAs, neurotrophins and other factors, such as miR-132, MeCP2, and BDNF, and broadened our understanding of how these neurotrophins and miRNAs are interconnected to regulate many physical and pathological processes. However, these areas are very new, and published results obtained from Medline are so few that conclusions based on these preliminary reports should always be drawn cautiously.

Nonetheless, promising findings are emerging from the initial series of studies and the notion that miRNAs and neurotrophins can direct therapeutic and diagnostic approaches. Notably, an inhibitor of miR-122 (miravirsen) is currently being tested in a phase $2 \mathrm{~A}$ clinical trial to assess safety and tolerability in treatment-naive patients with chronic hepatitis $C^{[76]}$. Studying the regulatory networks between neurotrophins and miRNAs might serve to find new targets for neurotrophic drugs ${ }^{[7]}$. To decrease the toxicity in normal cells, tumor-targeted nanoparticles have been developed and used to localize chemotherapeutics to tumors while enhancing efficacy ${ }^{[77]}$. Likewise, this strategy also can be used for the treatment of brain diseases by miRNAs or siRNAs to decrease the neurotoxicity from overloaded nucleic acids. For diagnostic approaches other than testing neurotrophin levels, miRNAs may provide novel diagnostic methods. Some miRNAs may be released through exosomes, as miR-16, and these secreted miRNAs are stable, transferable and functional in the recipient cells ${ }^{[78]}$, suggesting that specific miRNAs are promising biomarkers for cancer and brain diseases.

\section{References}

1 Segal RA. Selectivity in neurotrophin signaling: theme and variations. Annu Rev Neurosci 2003; 26: 299-330.

2 Reichardt LF. Neurotrophin-regulated signalling pathways. Philos Trans R Soc Lond B Biol Sci 2006; 361: 1545-64.

3 Allen SJ, Dawbarn D. Clinical relevance of the neurotrophins and their receptors. Clin Sci (Lond) 2006; 110: 175-91.

4 Chao MV, Rajagopal R, Lee FS. Neurotrophin signalling in health and disease. Clin Sci (Lond) 2006; 110: 167-73.

5 Park H, Poo MM. Neurotrophin regulation of neural circuit development and function. Nat Rev Neurosci 2013; 14: 7-23.

6 Underwood CK, Coulson EJ. The p75 neurotrophin receptor. Int J Biochem Cell Biol 2008; 40: 1664-8.

7 Longo FM, Massa SM. Small-molecule modulation of neurotrophin receptors: a strategy for the treatment of neurological disease. Nat Rev Drug Discov 2013; 12: 507-25.

8 Young KM, Merson TD, Sotthibundhu A, Coulson EJ, Bartlett PF. p75 neurotrophin receptor expression defines a population of BDNFresponsive neurogenic precursor cells. J Neurosci 2007; 27: 514655.

9 Galvao RP, Garcia-Verdugo JM, Alvarez-Buylla A. Brain-derived neurotrophic factor signaling does not stimulate subventricular zone neurogenesis in adult mice and rats. J Neurosci 2008; 28: 1336883.

10 Shi J, Longo FM, Massa SM. A small molecule p75(NTR) ligand protects neurogenesis after traumatic brain injury. Stem Cells 2013; 31: 2561-74.

11 Scardigli R, Capelli P, Vignone D, Brandi R, Ceci M, La Regina F, et al. Neutralization of nerve growth factor impairs proliferation and differentiation of adult neural progenitors in the subventricular zone. Stem Cells 2014; 32: 2516-28.

12 Numakawa T, Richards M, Adachi N, Kishi S, Kunugi H, Hashido K. MicroRNA function and neurotrophin BDNF. Neurochem Int 2011; 59 : 551-8.

13 Imam JS, Plyler JR, Bansal H, Prajapati S, Bansal S, Rebeles J, et al. Genomic loss of tumor suppressor miRNA-204 promotes cancer cell migration and invasion by activating AKT/mTOR/Rac1 signaling and actin reorganization. PLoS One 2012; 7: e52397.

14 Ambros V. The functions of animal microRNAs. Nature 2004; 431: 350-5.

15 Bartel DP. MicroRNAs: genomics, biogenesis, mechanism, and function. Cell 2004; 116: 281-97.

16 Almeida MI, Reis RM, Calin GA. MicroRNA history: discovery, recent applications, and next frontiers. Mutat Res 2011; 717: 1-8.

17 Filipowicz W, Bhattacharyya SN, Sonenberg N. Mechanisms of posttranscriptional regulation by microRNAs: are the answers in sight? Nat Rev Genet 2008; 9: 102-14.

18 Sandberg R, Neilson JR, Sarma A, Sharp PA, Burge CB. Proliferating cells express mRNAs with shortened $3^{\prime}$ untranslated regions and fewer microRNA target sites. Science 2008; 320: 1643-7.

19 Konopka W, Kiryk A, Novak M, Herwerth M, Parkitna JR, Wawrzyniak $\mathrm{M}$, et al. MicroRNA loss enhances learning and memory in mice. J Neurosci 2010; 30: 14835-42.

$20 \mathrm{Li} \mathrm{YJ,} \mathrm{Xu} \mathrm{M,} \mathrm{Gao} \mathrm{ZH,} \mathrm{Wang} \mathrm{YQ,} \mathrm{Yue} \mathrm{Z,} \mathrm{Zhang} \mathrm{YX,} \mathrm{et} \mathrm{al.} \mathrm{Alterations} \mathrm{of}$ serum levels of BDNF-related miRNAs in patients with depression. PLoS One 2013; 8: e63648.

21 Johannessen M, Delghandi MP, Moens U. What turns CREB on? Cell Signal 2004; 16: 1211-27.

22 Hong EJ, McCord AE, Greenberg ME. A biological function for the neuronal activity-dependent component of Bdnf transcription in the development of cortical inhibition. Neuron 2008; 60: 610-24.

23 Gao J, Wang WY, Mao YW, Graff J, Guan JS, Pan L, et al. A novel pathway regulates memory and plasticity via SIRT1 and miR-134. Nature 2010; 466: 1105-9.

24 Wang T, Liu YY, Wang X, Yang N, Zhu HB, Zuo PP. Protective effects of octacosanol on 6-hydroxydopamine-induced Parkinsonism in rats via regulation of ProNGF and NGF signaling. Acta Pharmacol Sin 2010; 31: 765-74.

25 Irmady K, Jackman KA, Padow VA, Shahani N, Martin LA, Cerchietti $\mathrm{L}$, et al. Mir-592 regulates the induction and cell death-promoting activity of p75NTR in neuronal ischemic injury. J Neurosci 2014; 34: 3419-28.

26 Risbud RM, Lee C, Porter BE. Neurotrophin-3 mRNA a putative target of miR21 following status epilepticus. Brain Res 2011; 1424: 53-9.

27 Othumpangat S, Walton C, Piedimonte G. MicroRNA-221 modulates RSV replication in human bronchial epithelium by targeting NGF expression. PLoS One 2012; 7: e30030.

28 Miura P, Amirouche A, Clow C, Belanger G, Jasmin BJ. Brainderived neurotrophic factor expression is repressed during myogenic differentiation by miR-206. J Neurochem 2012; 120: 230-8.

29 Guidi M, Muinos-Gimeno M, Kagerbauer B, Marti E, Estivill X, Espinosa-Parrilla $Y$. Overexpression of miR-128 specifically inhibits the truncated isoform of NTRK3 and upregulates BCL2 in SH-SY5Y 
neuroblastoma cells. BMC Mol Biol 2010; 11: 95.

30 Schratt GM, Nigh EA, Chen WG, Hu L, Greenberg ME. BDNF regulates the translation of a select group of mRNAs by a mammalian target of rapamycin-phosphatidylinositol 3-kinase-dependent pathway during neuronal development. J Neurosci 2004; 24: 7366-77.

31 Paroo Z, Ye X, Chen S, Liu Q. Phosphorylation of the human microRNA-generating complex mediates MAPK/Erk signaling. Cell 2009; 139: 112-22.

32 Huang YW, Ruiz CR, Eyler EC, Lin K, Meffert MK. Dual regulation of miRNA biogenesis generates target specificity in neurotrophin-induced protein synthesis. Cell 2012; 148: 933-46.

$33 \mathrm{Heo} \mathrm{I,} \mathrm{Joo} \mathrm{C,} \mathrm{Kim} \mathrm{YK,} \mathrm{Ha} \mathrm{M,} \mathrm{Yoon} \mathrm{MJ,} \mathrm{Cho} \mathrm{J,} \mathrm{et} \mathrm{al.} \mathrm{TUT4} \mathrm{in} \mathrm{concert}$ with Lin28 suppresses microRNA biogenesis through pre-microRNA uridylation. Cell 2009; 138: 696-708.

$34 \mathrm{Wu} \mathrm{J}, \mathrm{Xie} X$. Comparative sequence analysis reveals an intricate network among REST, CREB and miRNA in mediating neuronal gene expression. Genome Biol 2006; 7: R85.

35 Remenyi J, Hunter CJ, Cole C, Ando H, Impey S, Monk CE, et al. Regulation of the miR-212/132 locus by MSK1 and CREB in response to neurotrophins. Biochem J 2010; 428: 281-91.

36 Vo N, Klein ME, Varlamova O, Keller DM, Yamamoto T, Goodman RH, et al. A cAMP-response element binding protein-induced microRNA regulates neuronal morphogenesis. Proc Natl Acad Sci U S A 2005; 102: 16426-31.

37 Magill ST, Cambronne XA, Luikart BW, Lioy DT, Leighton BH, Westbrook $\mathrm{GL}$, et al. microRNA-132 regulates dendritic growth and arborization of newborn neurons in the adult hippocampus. Proc Natl Acad Sci U S A 2010; 107: 20382-7.

38 Laneve P, Gioia U, Andriotto A, Moretti F, Bozzoni I, Caffarelli E. A minicircuitry involving REST and CREB controls miR-9-2 expression during human neuronal differentiation. Nucleic Acids Res 2010; 38 : 6895-905.

39 Lukiw WJ. NF-kB, cyrillicB-regulated micro RNAs (miRNAs) in primary human brain cells. Exp Neurol 2012; 235: 484-90.

40 Ghose J, Sinha M, Das E, Jana NR, Bhattacharyya NP. Regulation of miR-146a by RelA/NFkB and p53 in STHdh(Q111)/Hdh(Q111) cells, a cell model of Huntington's disease. PLoS One 2011; 6: e23837.

41 Hu HY, Li KP, Wang XJ, Liu Y, Lu ZG, Dong RH, et al. Set9, NF-kappaB, and microRNA-21 mediate berberine-induced apoptosis of human multiple myeloma cells. Acta Pharmacol Sin 2013; 34: 157-66.

42 Terasawa K, Ichimura A, Sato F, Shimizu K, Tsujimoto G. Sustained activation of ERK1/2 by NGF induces microRNA-221 and 222 in PC12 cells. FEBS J 2009; 276: 3269-76.

43 Montalban E, Mattugini N, Ciarapica R, Provenzano C, Savino M, Scagnoli F, et al. MiR-21 is an Ngf-modulated microRNA that supports Ngf signaling and regulates neuronal degeneration in PC12 cells. Neuromolecular Med 2014; 16: 415-30.

44 Hamada N, Fujita Y, Kojima T, Kitamoto A, Akao Y, Nozawa Y, et al. MicroRNA expression profiling of NGF-treated PC12 cells revealed a critical role for miR-221 in neuronal differentiation. Neurochem Int 2012; 60: 743-50.

45 Meza-Sosa KF, Valle-Garcia D, Pedraza-Alva G, Perez-Martinez L. Role of microRNAs in central nervous system development and pathology. J Neurosci Res 2012; 90: 1-12.

46 Klein ME, Lioy DT, Ma L, Impey S, Mandel G, Goodman RH. Homeostatic regulation of MeCP2 expression by a CREB-induced microRNA. Nat Neurosci 2007; 10: 1513-4.

47 Packer AN, Xing Y, Harper SQ, Jones L, Davidson BL. The bifunctional microRNA miR-9/miR-9* regulates REST and CoREST and is downregulated in Huntington's disease. J Neurosci 2008; 28: 14341-6.

48 Johnson R, Zuccato C, Belyaev ND, Guest DJ, Cattaneo E, Buckley
NJ. A microRNA-based gene dysregulation pathway in Huntington's disease. Neurobiol Dis 2008; 29: 438-45.

49 Samadi P, Boutet A, Rymar V, Rawal K, Maheux J, Kvann JC, et al. Relationship between BDNF expression in major striatal afferents, striatum morphology and motor behavior in the R6/2 mouse model of Huntington's disease. Genes Brain Behav 2013; 12: 108-24.

50 Roux JC, Zala D, Panayotis N, Borges-Correia A, Saudou F, Villard L. Modification of Mecp2 dosage alters axonal transport through the Huntingtin/Hap1 pathway. Neurobiol Dis 2012; 45: 786-95.

51 Tan L, Yu JT, Tan L. Causes and consequences of microRNA dysregulation in neurodegenerative diseases. Mol Neurobiol 2014 Jun 29. [Epub ahead of print]

52 Lungu G, Stoica G, Ambrus A. MicroRNA profiling and the role of microRNA-132 in neurodegeneration using a rat model. Neurosci Lett 2013; 553: 153-8.

53 Nomura T, Kimura M, Horii T, Morita S, Soejima H, Kudo S, et al. MeCP2-dependent repression of an imprinted miR-184 released by depolarization. Hum Mol Genet 2008; 17: 1192-9.

54 Gehrke S, Imai Y, Sokol N, Lu B. Pathogenic LRRK2 negatively regulates microRNA-mediated translational repression. Nature 2010; 466: 637-41.

55 Yamakuchi M, Lowenstein CJ. MiR-34, SIRT1 and p53: the feedback loop. Cell Cycle 2009; 8: 712-5.

56 Lusardi TA, Farr CD, Faulkner CL, Pignataro G, Yang T, Lan J, et al. Ischemic preconditioning regulates expression of microRNAs and a predicted target, MeCP2, in mouse cortex. J Cereb Blood Flow Metab 2010; 30: 744-56.

57 Pulliero A, Fazzi E, Cartiglia C, Orcesi S, Balottin U, Uggetti C, et al. The Aicardi-Goutieres syndrome. Molecular and clinical features of RNAse deficiency and microRNA overload. Mutat Res 2011; 717: 99-108.

58 Mellios N, Huang HS, Grigorenko A, Rogaev E, Akbarian S. A set of differentially expressed miRNAs, including miR-30a-5p, act as posttranscriptional inhibitors of BDNF in prefrontal cortex. Hum Mol Genet 2008; 17: 3030-42.

59 Croce N, Gelfo F, Ciotti MT, Federici G, Caltagirone C, Bernardini S, et al. NPY modulates miR-30a-5p and BDNF in opposite direction in an in vitro model of Alzheimer disease: a possible role in neuroprotection? Mol Cell Biochem 2013; 376: 189-95.

60 Au CW, Siu MK, Liao X, Wong ES, Ngan HY, Tam KF, et al. Tyrosine kinase $B$ receptor and BDNF expression in ovarian cancers - effect on cell migration, angiogenesis and clinical outcome. Cancer Lett 2009; 281: 151-61.

61 Brodeur GM. Neuroblastoma: biological insights into a clinical enigma. Nat Rev Cancer 2003; 3: 203-16.

62 Edsjo A, Lavenius E, Nilsson H, Hoehner JC, Simonsson P, Culp LA, et al. Expression of trkB in human neuroblastoma in relation to MYCN expression and retinoic acid treatment. Lab Invest 2003; 83: 81323.

63 Nakagawara A, Azar CG, Scavarda NJ, Brodeur GM. Expression and function of TRK-B and BDNF in human neuroblastomas. Mol Cell Biol 1994; 14: 759-67.

64 Howe EN, Cochrane DR, Cittelly DM, Richer JK. miR-200c targets a NF-kappaB up-regulated TrkB/NTF3 autocrine signaling loop to enhance anoikis sensitivity in triple negative breast cancer. PLoS One 2012; 7: e49987.

65 Laneve P, Di Marcotullio L, Gioia U, Fiori ME, Ferretti E, Gulino A, et al. The interplay between microRNAs and the neurotrophin receptor tropomyosin-related kinase $\mathrm{C}$ controls proliferation of human neuroblastoma cells. Proc Natl Acad Sci U S A 2007; 104: 7957-62.

66 Vilar M, Charalampopoulos I, Kenchappa RS, Reversi A, Klos- 
Applequist JM, Karaca E, et al. Ligand-independent signaling by disulfide-crosslinked dimers of the p75 neurotrophin receptor. J Cell Sci 2009; 122: 3351-7.

67 Parsi S, Soltani BM, Hosseini E, Tousi SE, Mowla SJ. Experimental verification of a predicted intronic microRNA in human NGFR gene with a potential pro-apoptotic function. PLoS One 2012; 7: e35561.

68 Dizaj SM, Jafari S, Khosroushahi AY. A sight on the current nanoparticle-based gene delivery vectors. Nanoscale Res Lett 2014; 9: 252.

69 Pulliero A, Marengo B, Fenoglio D, Parodi A, Cereda C, Domenicotti $\mathrm{C}$, et al. Prevention of lymphocyte neurotoxic effects by microRNA delivery. MicroRNA 2014; 2: 187-93.

70 Danhier F, Ansorena E, Silva JM, Coco R, Le Breton A, Preat V. PLGAbased nanoparticles: an overview of biomedical applications. J Control Release 2012; 161: 505-22.

71 Sharma D, Maheshwari D, Philip G, Rana R, Bhatia S, Singh M, et al. Formulation and optimization of polymeric nanoparticles for intranasal delivery of lorazepam using Box-Behnken design: in vitro and in vivo evaluation. Biomed Res Int 2014; 156010.

72 Sankar R, Ravikumar V. Biocompatibility and biodistribution of suberoylanilide hydroxamic acid loaded poly ( $D L$-lactide-co-glycolide) nanoparticles for targeted drug delivery in cancer. Biomed Pharmacother. 2014 Jul 17. doi: 10.1016/j.biopha.2014.07.015.

73 Jensen SA, Day ES, Ko CH, Hurley LA, Luciano JP, Kouri FM, et al. Spherical nucleic acid nanoparticle conjugates as an RNAi-based therapy for glioblastoma. Sci TransI Med 2013; 5: 209 ra152.

74 Arora S, Swaminathan SK, Kirtane A, Srivastava SK, Bhardwaj A,
Singh S, et al. Synthesis, characterization, and evaluation of poly $(D, L-$ lactide-co-glycolide)-based nanoformulation of miRNA-150: potential implications for pancreatic cancer therapy. Int J Nanomed 2014; 9: 2933-42.

75 Tiwari SK, Agarwal S, Seth B, Yadav A, Nair S, Bhatnagar P, et al. Curcumin-loaded nanoparticles potently induce adult neurogenesis and reverse cognitive deficits in Alzheimer's disease model via canonical Wnt/beta-catenin pathway. ACS Nano 2014; 8: 76-103.

76 Janssen HL, Reesink HW, Lawitz EJ, Zeuzem S, Rodriguez-Torres M, Patel K, et al. Treatment of HCV infection by targeting microRNA. N Engl J Med 2013; 368: 1685-94.

77 Mueller M, Reichardt W, Koerner J, Groettrup M. Coencapsulation of tumor lysate and CpG-ODN in PLGA-microspheres enables successful immunotherapy of prostate carcinoma in TRAMP mice. J Control Release 2012; 162: 159-66.

78 Kosaka N, Iguchi H, Yoshioka Y, Takeshita F, Matsuki Y, Ochiya T. Secretory mechanisms and intercellular transfer of microRNAs in living cells. J Biol Chem 2010; 285: 17442-52.

This work is licensed under the Creative Commons Attribution-NonCommercial-No Derivative Works 3.0 Unported License. To view a copy of this license, visit http://creativecommons.org/licenses/ by-nc-nd/3.0/ 